\title{
Soft-patchy nanoparticles: modeling and self-organization
}

\author{
Emanuela Bianchi, ${ }^{\text {a }}$ Barbara Capone, ${ }^{\mathrm{b}}$ Gerhard Kahl ${ }^{\mathrm{c}}$ \\ and Christos N. Likos ${ }^{\mathrm{b}}$
}

Received 19th December 2014, Accepted 15th January 2015

DOI: $10.1039 / \mathrm{c} 4 \mathrm{fd} 00271 \mathrm{~g}$

We consider a novel class of patchy particles inspired by polymer-based complex units where the limited valence in bonding is accompanied by soft interactions and incessant fluctuations of the patch positions, possibly leading to reversible modifications of the patch number and size. We introduce a simple model that takes into account the aforementioned features and we focus on the role played by the patch flexibility on the self-organization of our patchy units in the bulk, with particular attention to the connectivity properties and the morphology of the aggregated networks.

\section{Introduction}

Self-organization at the atomic or molecular level is a fundamental mechanism of paramount relevance in nature. During the past years this mechanism has been exploited increasingly more frequently in materials science to fabricate target mesoscopic structures for technological applications. ${ }^{1}$ A successful way to produce materials with well-defined architectures and properties often relies on anisotropy-driven self-organization. ${ }^{2}$ At the nano- or micro-scale level, interparticle interactions can indeed be designed to be anisotropic, for instance by creating patterns on the surface of the self-organizing entities: surface regions with chemical or physical properties different from those of the bare colloid are usually referred to as patches. By virtue of their directional and selective bonding capacities, patchy ${ }^{3,4}$ and inverse patchy ${ }^{5}$ particles, i.e., patchy particles with charged patches, can assemble in a large variety of mesoscopic structures., ${ }^{\mathbf{3 , 4}, \mathbf{1 3}}$

In most of the patchy and inverse patchy systems investigated so far, the accurate design of the particle surface pattern (in terms of position, shape and extent of the patches) has been a key ingredient for the assembly of the desired mesoscopic architectures. When some patch rearrangement was allowed, units

anstitut für Theoretische Physik, Technische Universität Wien, Wiedner Hauptstraße 8-10, A-1040 Vienna, Austria.E-mail: emanuela.bianchi@tuwien.ac.at

${ }^{b}$ Faculty of Physics, University of Vienna, Boltzmanngasse 5, A-1090, Vienna, Austria

'Institut für Theoretische Physik and Center for Computational Materials Science (CMS), Technische Universität Wien, Wiedner Hauptstraße 8-10, A-1040 Vienna, Austria 
with reduced bonding valence were shown to favor disordered phases and stabilize the liquid with respect to the crystal in the zero-temperature limit. ${ }^{\mathbf{1 4}}$ Additionally, as soon as patchy particles with a low number of mobile, directional bonds were designed to interact in a soft fashion, the solid-liquid phase separation was suppressed in favor of a coexistence between two disordered phases: a single and a double network, the latter consisting of two inter-penetrating, noninteracting single networks. ${ }^{15}$ Patchy systems combining low valence, bond flexibility and soft interactions were designed to neglect double bonding between patches as well as possible energy penalties associated with the distortion of the equilibrium patch arrangement. These features can, for instance, be realized in associating fluids or DNA-coated colloid systems. On the other side of the spectrum, tetravalent DNA nanostars, i.e., extremely flexible patchy units with four bonding patches, have been shown to never crystallize and form instead a thermodynamically stable, fully bonded equilibrium gel. ${ }^{16,17}$

In the present contribution we introduce a class of patchy particles where directional bonding, soft interactions, and incessant patch rearrangements are complemented with energy penalties associated with the mobility of the patches, reversible modifications of the patch number/size and the possible formation of more than two bonds per patch. Our model is inspired by deformable patchy units that self-assemble in a bulk system of star polymers with functionalized arms. Such functionalized star polymers, also known as telechelic star polymers (TSPs), are macromolecules made of diblock polymeric chains grafted on a common center in a selective solvent: each arm has a solvophilic part, which is located in the interior of the star, and an attractive, solvophobic end. The propensity of the solvophobic ends of different arms to associate gives rise to a number of particular intra-star and inter-star association phenomena. ${ }^{18-20}$ In particular, it was recently shown that the functionalized ends of the arms self-organize into soft, attractive patches, while the non-functionalized parts of the arms act as ultra-soft, repulsive cores: $:^{21,22}$ in the zero density limit, the number of patches per star and the equilibrium patch positions can be controlled by changing the percentage of attractive monomers per arm and the number of arms per star; at finite density, the intra-star association, which is faster than the inter-star association, leads to systems composed of equilibrium patchy aggregates, which maintain the same number of patches as their zero-density counterparts. These self-assembled, polymer-based patchy units are thus equilibrium structures that can interact with each other while keeping, on average, a well-defined internal structure. The possibility of tuning the number and the equilibrium arrangement of the patches, in combination with the capability of these patchy assemblies to maintain their internal structure at finite density, strongly motivates the investigation of these systems in the bulk. In this contribution we consider a simple patchy model that keeps a close connection to the described polymer-based complex units, while dealing with interaction potentials that are computationally easier to handle. Our aim is to explore the role of the softness and of the patch rearrangement on the formation of particle networks at a coarse-grained level where both physical features can be controlled by suitably chosen parameters.

Our soft and flexible patchy colloid (SFPC) model features a soft, repulsive core on which a fixed number of soft, attractive patches is attached. Both the patchcore distance and the patch-patch angle within the same particle are allowed to fluctuate around their respective equilibrium values. The movements of the 
patches are controlled by two flexibility parameters: one describes the patch elongation along the radial direction, while the other is associated with the angular patch freedom. The internal energy of each patchy unit is the result of pair interactions occurring between the single entities - either cores or patches belonging either to the same particle or to two different particles. The functional form and the specific values of the parameters of the pair interaction are chosen in such a way that, in the limit of non-flexible patches, our model can be related to a rigid counterpart whose phase diagram is reported in the literature. ${ }^{23}$

In the present contribution we explore by means of Monte Carlo (MC) simulations the bulk behavior of the fluid phase at a fixed density; this value corresponds to the density at which the rigid reference model forms a fully bonded, homogeneous phase with an open architecture; different temperatures (or interaction strengths) are considered. We put particular focus on the impact of the patch flexibility on the features of the percolating structures formed in our SFPC systems.

The manuscript is organized as follows: in Section 2 we describe our SFPC model, in Section 3 we provide details of the MC simulations, in Section 4 we report our results, and in Section 5 we present our conclusions.

\section{The model}

Recent studies on TSPs showed that systems with an average spherical repulsive core and functionalized sites, flexibly distributed around the core, allow the mechanical stabilization of crystals over a portion of the phase diagram that is one order of magnitude wider than in systems with the same particle valence where the patches are fixed in rigid geometries on the particle surface. ${ }^{21}$ At the same time, the occurrence of gel states in TSP systems has also been observed. ${ }^{22}$ It is thus important to analyze the effect of the patch flexibility on the selforganization of patchy units into extended structures. Starting from the fully rigid reference system, ${ }^{23}$ we hereby introduce suitable potentials characterized by two specific flexibility parameters that allow for angular and radial displacements of the patches with respect to the core of the units they belong to.

A soft and flexible patchy colloid (SFPC) consists of a fixed number of mutually attractive spheres, referred to as patches (index "p"), connected to the center of a soft repulsive sphere, referred to as the core (index "c"). A schematic representation of such a patchy unit is reported in Fig. 1 for the four patch case. The internal energy of a collection of SFPCs can be split into two distinct contributions: the interparticle (or mutual) interaction and the intra-particle (or self) energy.

\subsection{Inter-particle interactions}

The inter-particle or mutual interaction is the sum of three contributions: the attraction between the patches (index "pp"), the repulsion between the cores (index "cc"), and the patch-core repulsion (index "pc"); the entities (either patches or cores) involved in these pair interactions belong to different SFPCs.

We consider a $2 n-n$ potential for the attractive pp interaction:

$$
V_{\mathrm{pp}}^{\mathrm{m}}\left(r_{\mathrm{pp}}\right)=4 \varepsilon_{\mathrm{pp}}\left[\left(\sigma_{\mathrm{pp}} / r_{\mathrm{pp}}\right)^{2 n}-\left(\sigma_{\mathrm{pp}} / r_{\mathrm{pp}}\right)^{n}\right]
$$




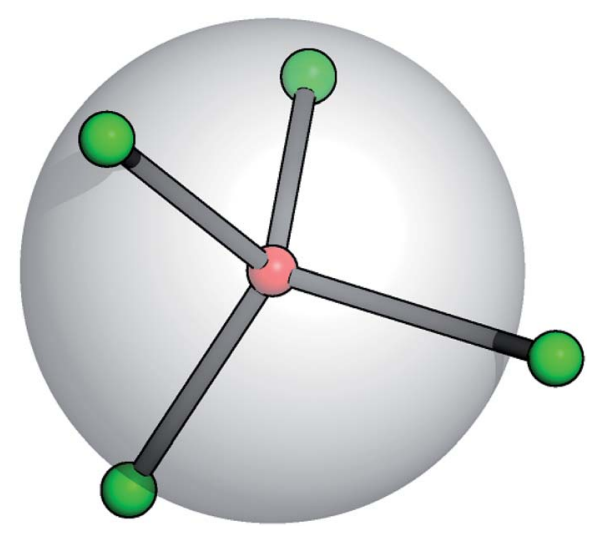

Fig. 1 Schematic representation of a soft and flexible patchy colloid (SFPC) with four patches. The big gray sphere features the soft particle core, the small green spheres represent the patches, and the central red sphere represents the anchor point to which the patches are linked. Patch displacements are allowed in radial and tangential directions. The sticks are guides to the eye.

and a Lennard-Jones potential for the repulsive cc and pc interactions:

$$
V_{\mathrm{ij}}^{\mathrm{m}}\left(r_{\mathrm{ij}}\right)=\left\{\begin{array}{l}
4 \varepsilon_{\mathrm{ij}}\left[\left(\sigma_{\mathrm{ij}} / r_{\mathrm{ij}}\right)^{12}-\left(\sigma_{\mathrm{ij}} / r_{\mathrm{ij}}\right)^{6}\right] \text { when } r_{\mathrm{ij}} \leq \sigma_{\mathrm{ij}} \\
0 \text { otherwise }
\end{array}\right.
$$

where $\mathrm{ij}=\mathrm{cc}$ or pc. The parameters of these three potentials are set such that the correspondence with the reference rigid patchy model is guaranteed. ${ }^{23}$ In particular, we choose $\sigma_{\mathrm{cc}}$ as our length unit; we further choose $\sigma_{\mathrm{pc}}=0.5$ and $\sigma_{\mathrm{pp}}=$ 0.05. All energy strengths are fixed to the same unit value, i.e. $\varepsilon_{\mathrm{cc}}=\varepsilon_{\mathrm{pc}}=\varepsilon_{\mathrm{pp}}=1$. Finally, we need to set $n$ such that the patch-patch attraction behaves in a similar manner as compared to its counterpart in the rigid reference model: ${ }^{23}$ the attraction must be sufficiently long-ranged and must have a minimum where the core-core distance between two patchy units is $r_{\mathrm{cc}}=1.07177 ;^{23}$ in this way the optimal spacing of the spatially extended open structure is preserved going from the rigid to the flexible model. When two patches on different SFPCs are positioned at the equilibrium distance from their core and are perfectly aligned along the vector connecting the two particle centers, the mapping to the rigid model is satisfied for $n=2$. In panels (a)-(c) of Fig. 2, we report the resulting potentials for selected elastic constants.

\subsection{Intra-particle interactions}

The intra-particle or self energy is the sum of the interactions within a single SFPC. The patch-patch interaction consists of a distance-dependent, attractive part, expressed by the same patch-patch potential described above, i.e. $V_{\mathrm{pp}}^{\mathrm{s}}\left(r_{\mathrm{pp}}\right)=$ $V_{\mathrm{pp}}^{\mathrm{m}}\left(r_{\mathrm{pp}}\right)$, and an angular constraint $S_{\mathrm{pp}}^{\mathrm{s}}(\theta)$ which prevents the patches on the same particle from collapsing on top of each other. This constraint is an efficient way for capturing the self-avoiding behavior of the branches of the functionalized star polymers that represent the basis of our SFPC model. The functional form of such a constraint is a generalized spring potential with an average value equal to the 

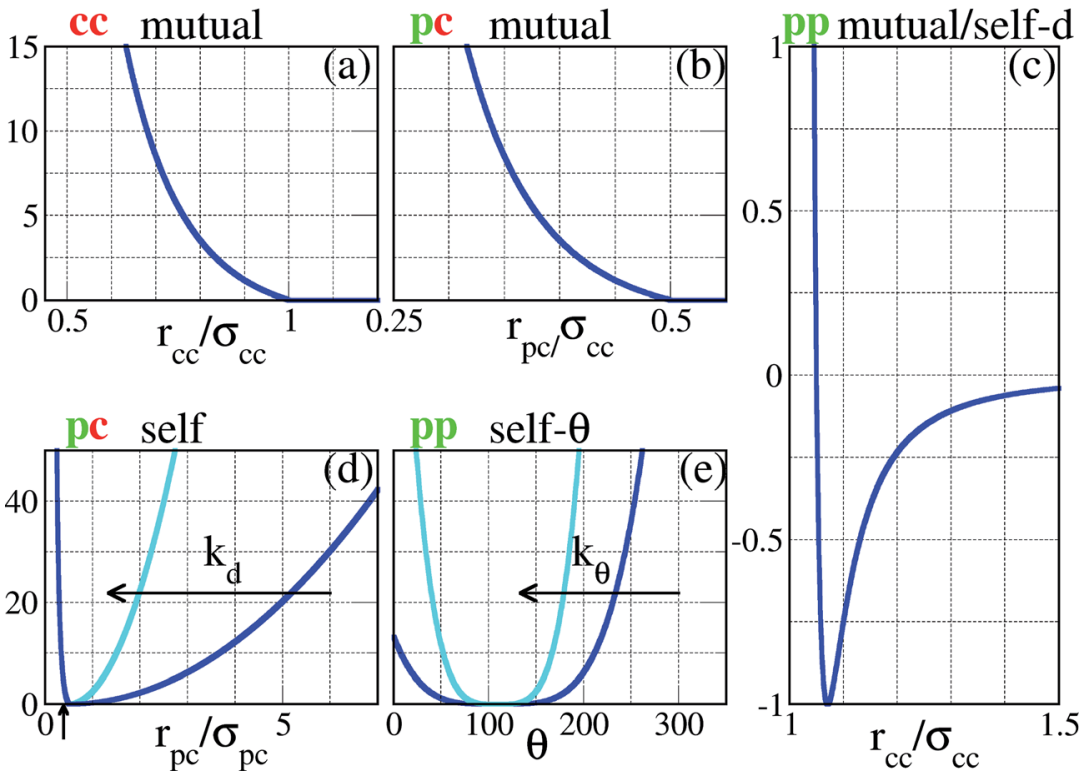

Fig. 2 Representation of the interaction potentials between the different components of our SFPCs (the vertical axes correspond to energies, measured in units of $\varepsilon_{\mathrm{pp}}$ ). In panels $(\mathrm{a})-(\mathrm{c})$, the core-core, the patch-core and the patch-patch potentials between entities belonging to two distinct SFPCs are reported (labeled as cc mutual, pc mutual and pp mutual, respectively). In panels (c)-(e), the radius-dependent patch-patch, the patchcore and the angle-dependent patch-patch potentials between entities belonging to the same SFPC are reported (labeled as pp self- $d$, pc self, and pp self- $\theta$, respectively). To facilitate the comparison with the pair potential of the rigid system reported in ref. 23 , the pp mutual potential is reported versus the distance between the SFPC cores, assuming that the patch-core distance is exactly $\sigma_{\mathrm{pc}}$; the pp self- $d$ potential must thus be shifted by a distance $\sigma_{\mathrm{pc}}$ in order to visualize it as a function of the distance between two patches on the same patchy units. The $\mathrm{pc}$ self and the pp self- $\theta$ potentials represent the constraints on the patch mobility; the flexibility of the patchy unit is described via two parameters, namely $k_{\mathrm{d}}$, responsible for the patch elongation along radial directions, and $k_{\theta}$, related to the angular patch freedom; in the figure the cases $k_{\mathrm{d}}=k_{\theta}=1$ (blue) and 10 (turquoise) are depicted.

equilibrium angle of a regular patch decoration, i.e. $\theta_{\mathrm{pp}}^{0}=109.5^{\circ}$ in the case of four patches, arranged on a regular tetrahedron; the functional form is given by

$$
S_{\mathrm{pp}}^{\mathrm{s}}(\theta)=k_{\theta}\left(\theta-\theta_{\mathrm{pp}}^{0}\right)^{4},
$$

where $k_{\theta}$ is the flexibility parameter that specifies the strength of the above potential.

The patch-core interaction consists of a repulsive part, expressed by the same patch-core potential described above, i.e. $V_{\mathrm{pc}}^{\mathrm{s}}\left(r_{\mathrm{pp}}\right)=V_{\mathrm{pc}}^{\mathrm{m}}\left(r_{\mathrm{pp}}\right)$, plus an attractive constraint $S_{\mathrm{pc}}^{\mathrm{s}}\left(r_{\mathrm{pc}}\right)$ which keeps the patches attached to their respective particle core. The equilibrium patch-core position is $\sigma_{\mathrm{pc}}$ : when a patch is closer than $\sigma_{\mathrm{pc}}$ to its core then it feels the repulsion, while when the patch is farther than $\sigma_{\mathrm{pc}}$ from its core it feels the attraction. The fluctuations around the equilibrium position are again described by a spring potential, namely 


$$
S_{\mathrm{pc}}^{\mathrm{s}}\left(r_{\mathrm{pc}}\right)=k_{\mathrm{d}}\left(r_{\mathrm{pc}}-\sigma_{\mathrm{pc}}\right)^{2},
$$

where $k_{\mathrm{d}}$ is the flexibility parameter that specifies the strength of the above potential. In panels (c)-(e) of Fig. 2 we report all contributions to the self energy for selected elastic constants.

\section{Monte Carlo simulations}

We perform Monte Carlo (MC) simulations in the NVT ensemble, where $N=512$ is the number of patchy units, $V=970.299$ is the volume of the simulations box in units of ${\sigma_{\mathrm{cc}}}^{3}$, and $T$ is the temperature in units of $\varepsilon_{\mathrm{pp}}$ (with $k_{\mathrm{B}}=1$ ). It is worth noting that in our simulations, while $T$ is varied, the number density of the systems is fixed to $\rho \approx 0.5277$ in units of $\sigma_{\mathrm{cc}}{ }^{-3}$; this choice corresponds to the density at which the rigid reference system ${ }^{23}$ is able to form a fully bonded, homogeneous and open structure-namely the diamond cubic lattice-which spans the whole simulation box. ${ }^{23}$

Each MC move consists, on average, of $N$ trial displacements; in order to take into account the internal degrees of freedom of our SFPCs, $\sim 50 \%$ of the trial displacements are particle moves, while $\sim 50 \%$ are patch displacements. An intraparticle move is defined as a rigid displacement of the whole SFPC by a random quantity distributed uniformly between $\pm \delta r_{\mathrm{m}}$; an inter-particle move is defined as a displacement of a single patch by a random quantity distributed uniformly between $\pm \delta r_{\mathrm{s}}$. The choice of the maximum amount of these displacements keeps an acceptance ratio of the corresponding move between $30 \%$ and $40 \%$.

In the present contribution, we focus on the role played by the patch flexibility on the self-organization of SFPCs in the bulk; we thus consider a selection of systems characterized by different sets of $\left(k_{\mathrm{d}}, k_{\theta}\right)$-values at three different temperatures, namely $T=0.10,0.30$ and 0.70 . We select a grid of flexibility parameters on a logarithmic scale: both $k_{\mathrm{d}}$ and $k_{\theta}$ range from 1 to 1000 in units of $\varepsilon_{\mathrm{pp}} / \sigma_{\mathrm{cc}}{ }^{2}$ and $\varepsilon_{\mathrm{pp}} \mathrm{rad}^{-4}$, respectively. We initialize all the simulations in the fluid phase and analyze the morphology of the system using the respective final configuration of the MC simulation. The shortest MC simulations (corresponding to systems in the fluid phase) extend over at least $10^{6} \mathrm{MC}$ steps, while the longest simulations (corresponding to systems in the gel states) cover $10^{7} \mathrm{MC}$ steps. Gels observed in the selected SFPC systems are out of equilibrium structures that show a small annealing even after long equilibration times.

We characterize the morphology of the systems via different quantities, namely the intra-particle and the inter-particle energies, the number of bonds formed between patches either on different particles (mutual bonds) or on the same particle (self bonds), the average angle between patches linked to the same core, and the average distance between a patch and its core. Finally, we also evaluate if the system is percolating or not; our percolation analysis is based on a minimum energy criterion: we consider two patches to be bonded when their interaction energy amounts to less than $10 \% \varepsilon_{\mathrm{pp}}$.

\section{Results}

As observed in the previous studies on TSPs, ${ }^{21,22}$ soft and flexible patchy units tend to form percolating gel networks; we thus expect to observe similar behavior in 
our SFPC systems. For TSPs the radial and angular flexibilities, as well as the attraction strength between the patches, depend on the microscopic details of the underlying systems and are consequently coupled to each other. In contrast, with our SFPC model we are able to investigate separately the role of the radial and angular flexibilities, on one side, and the effect of a change in the attraction strength (or equivalently, a change in temperature), on the other side. In particular, we aim to describe how the morphology of the self-organized structures depends on the flexibility parameters $k_{\mathrm{d}}$ and $k_{\theta}$ at three selected $T$-values (namely, $T=0.10,0.30$ and 0.70 ) and at a fixed density (namely, $\rho \approx 0.5277$ ).

In order to classify the self-organized structures, we first characterize the single particle architecture by considering two distinct features: the average distance between the patches and their corresponding core, $d_{\mathrm{pc}}$, and the average angle between two patches belonging to the same unit, $\Theta_{\mathrm{pp}}$. In Fig. 3, both quantities are represented at fixed temperature in contour plots in the $k_{\mathrm{d}} v s . k_{\theta}$ plane; data for all selected systems are reported for the three investigated temperatures. As expected, while the average patch-core distance depends mainly on $k_{\mathrm{d}}$, fluctuations of the average patch-patch angle are predominantly influenced by $k_{\theta}$. On lowering the temperature, both sets of data show rapidly diminishing deviations from their respective equilibrium values, i.e., both $\left(d_{\mathrm{pc}}-\sigma_{\mathrm{pc}}\right)$ and $\left(\Theta_{\mathrm{pp}}-\theta_{\mathrm{pc}}^{0}\right)$ grow with $T$. In particular, while at the highest temperature investigated, $d_{\mathrm{pc}}$ attains values of almost $3 \sigma_{\mathrm{pc}}$ for $k_{\mathrm{d}}<10$; it is on average less than $2 \sigma_{\mathrm{pc}}$ for the corresponding cases at the lowest temperature considered. In a similar way, in systems with $k_{\theta}<10, \Theta_{\mathrm{pp}}$ is smaller than $\theta_{\mathrm{pp}}^{0}$ by about $2^{\circ}$ at $T=0.10$, while it is about $10^{\circ}$ smaller than $\theta_{\mathrm{pp}}^{\mathrm{O}}$ at $T=0.70$.

The trends of the single particle structural features with temperature have two consequences. First of all, the increase of the patch-core distance enhances the effective packing fraction of the system: as $d_{\mathrm{pc}}$ varies from $0.5 \sigma_{\mathrm{cc}}$ (in the most rigid systems at the lowest temperature) to almost $1.5 \sigma_{\mathrm{cc}}$ (in the most flexible cases at the highest temperature), the packing fraction becomes seven times bigger. Moreover, the increase of both the radial and the angular patch fluctuations leads to effectively larger bonding volumes, thus favoring the formation of more than one bond per patch. The changes of the effective packing fraction of the system and of the bonding volume per particle have an impact on the inter-particle bonding, as demonstrated in the following.

To classify the different self-organized morphologies emerging in SFPC systems, we consider the average inter-particle (or mutual) energy, $e_{\mathrm{m}}$, the corresponding average number of bonds between patches belonging to different particles (or mutual bonds), $b_{\mathrm{m}}$, and the percolation threshold, signaled by the presence of at least one cluster that extends over the entire simulation box.

At $T=0.10$, the rigid reference system is able to form a fully bonded diamond crystal, where the average energy per particle, due to inter-particle bonding, is $\approx-1.65 .^{23}$ In contrast, at $T=0.30$ and 0.70 , the system is in the fluid state, characterized by an almost vanishing inter-particle energy. It must be noted that, even though the diamond crystal is the thermodynamically stable phase, when the system is initialized in the fluid phase and instantaneously quenched at $T=$ 0.10 (the same procedure that we use for our SFPCs), particles self-organize into a disordered percolating structure, where the average inter-particle energy is $\approx-0.96$. In other words, while the number of bonds of a rigid patchy particle in 

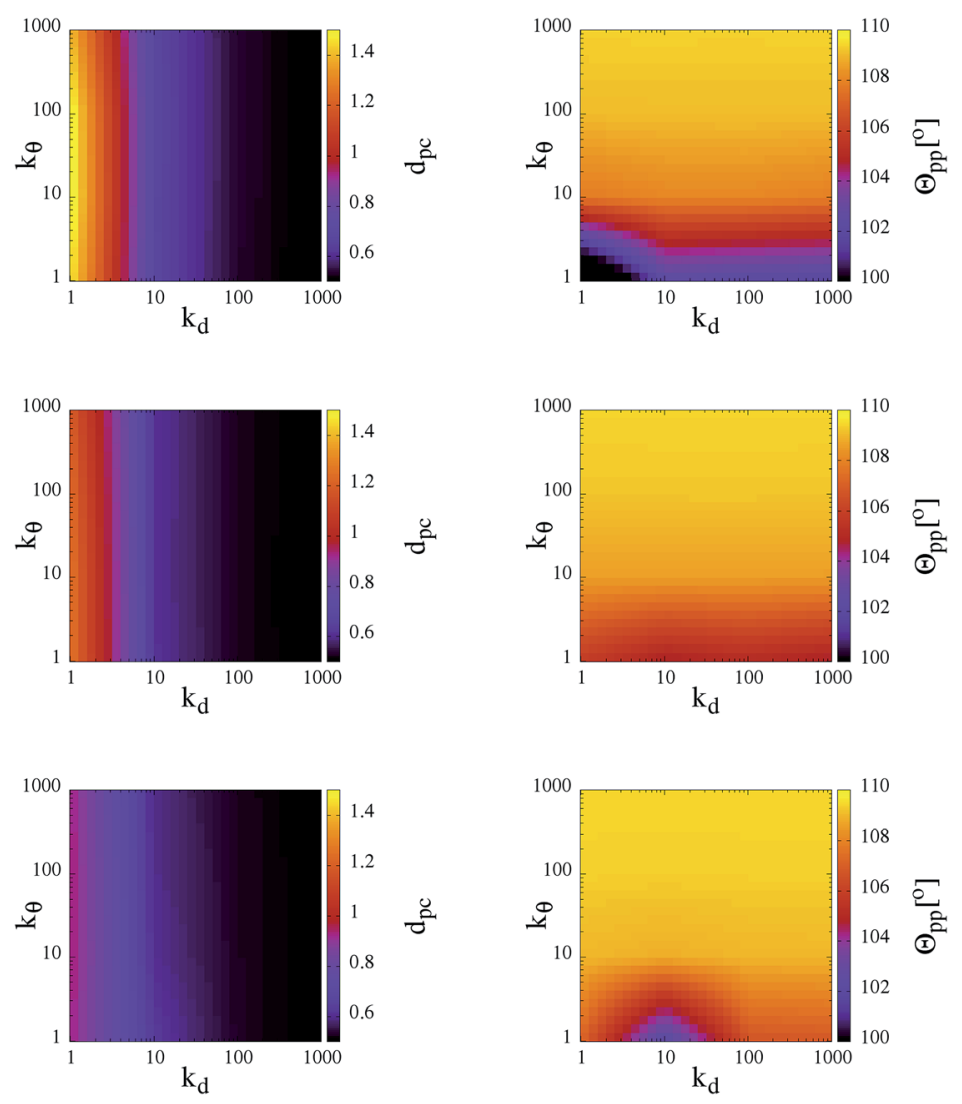

Fig. 3 Single particle structural features of the SFPCs: average patch-core distance $d_{\mathrm{pc}}$ (left column) and average patch-patch angle $\Theta_{\mathrm{pp}}$ (right column) between entities belonging to the same unit. Data are reported as contour plots in the $k_{\mathrm{d}} v s$. $k_{\theta}$ plane for three temperatures: $T=0.70,0.30$ and 0.10 (from top to bottom). Color scales are given at the right-hand sides of the panels. Data, represented by colors, are interpolated between the values on the grid specified by $k_{\mathrm{d}}, k_{\theta}=1,10,100$ and 1000 in their respective reduced units.

the lattice is four, in the disordered network not all patches are bonded and thus a rigid patchy particle in the gel state at $T=0.10$ forms less than four bonds.

In Fig. 4 , the corresponding data for $e_{\mathrm{m}}$ and $b_{\mathrm{m}}$ for our SFPCs are reported as contour plots in the $k_{\mathrm{d}} v s . k_{\theta}$ plane for all selected systems; again, each panel in the figure corresponds to one temperature. Similar to the rigid case, $e_{\mathrm{m}}$ and $b_{\mathrm{m}}$ provide a consistent picture of the system: when the system is in the fluid phase $e_{\mathrm{m}} \approx 0$, then also $b_{\mathrm{m}} \approx 0$; in contrast, extended bonded structures are characterized by $e_{\mathrm{m}}$ $\ll 0$, or equivalently, by $b_{\mathrm{m}} \gg 0$. While at $T=0.10$ particles self-organize into extended gel networks over the entire $\left(k_{\mathrm{d}}, k_{\theta}\right)$-range, for $T>0.10$ two different scenarios can be identified according to the radial flexibility: for $k_{\mathrm{d}} \geq 10$ the systems are in the fluid phase irrespective of the value of $k_{\theta}$, while for $k_{\mathrm{d}}<10$ the systems form deeply inter-connected networks over the entire $k_{\theta}$ range. The data provide evidence that the transition between the two scenarios at $k_{\mathrm{d}} \simeq 10$ is rather well-defined. Such a sharp distinction suggests that $k_{\mathrm{d}}$ is the key parameter that 

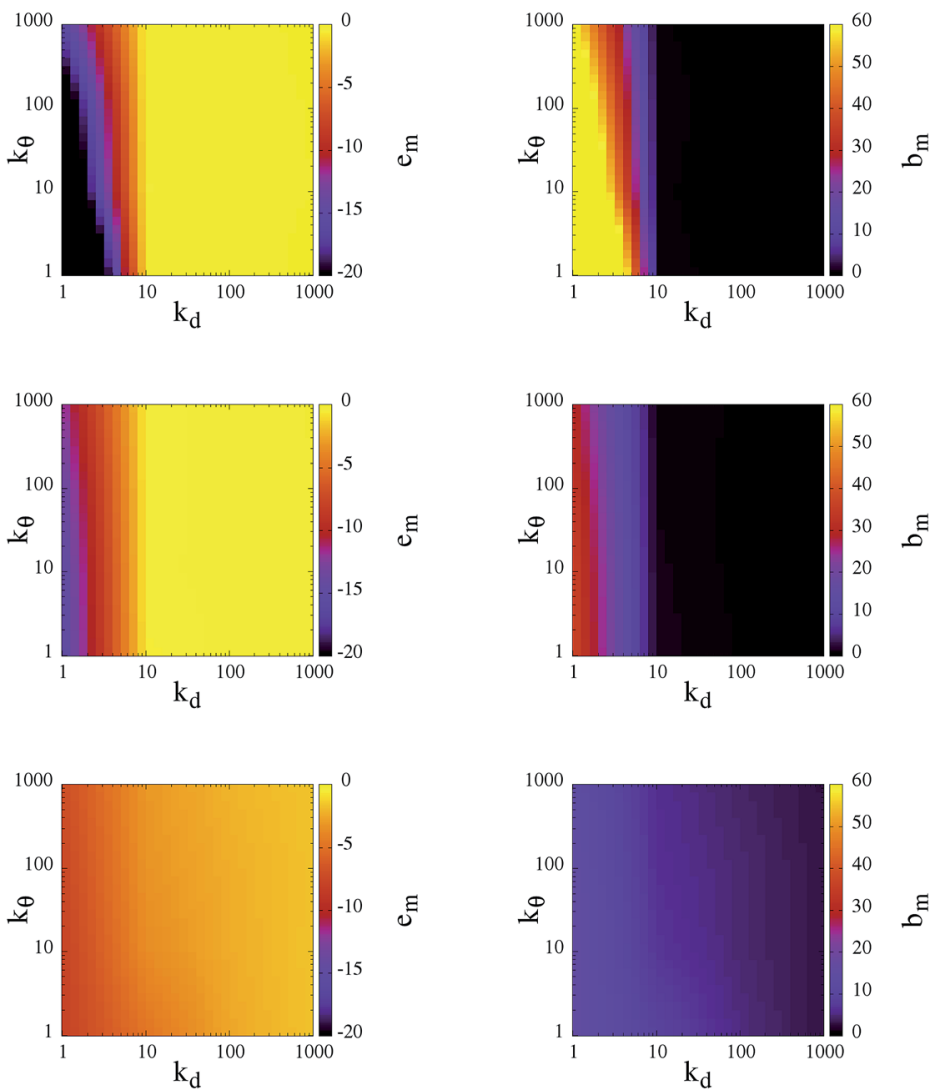

Fig. 4 Left column: inter-particle (mutual) energy per particle, $e_{m}$; right column: number of inter-particle (mutual) bonds per particle, $b_{\mathrm{m}}$. Data are reported as contour plots in the $k_{\mathrm{d}}$ vs. $k_{\theta}$ plane for three temperatures: $T=0.70,0.30$ and 0.10 (from top to bottom). Color scales are given at the right-hand sides of the panels. Data, represented by colors, are interpolated between the values on the grid specified by $k_{d}, k_{\theta}=1,10,100$ and 1000 in their respective reduced units.

governs the self-organization of our SFPCs; thus it can be used to distinguish between percolating and fluid states at intermediate and high temperatures. By softening the radial localization, i.e., by decreasing $k_{\mathrm{d}}$, the particle interaction range is indeed effectively increased (or, as mentioned before, the effective packing fraction is augmented), and thus even particles that are farther away from each other are able to form bonds. The angular flexibility starts only to play a role at the highest temperature investigated: the softer the angular localization is, the more interconnected the system is. All branched networks are found to be percolating.

Different characteristic features in the gel structure can be observed at different state points. In contrast to rigid patchy colloids, the number of bonds that a patch forms can be greater than one in SFPC systems: as mentioned before, the bonding volume increases together with either the temperature or the (radial and angular) flexibility. At the lowest temperature investigated, the differences in $e_{\mathrm{m}}$ and $b_{\mathrm{m}}$ are small for the chosen $\left(k_{\mathrm{d}}, k_{\theta}\right)$-value; nonetheless, the emerging percolating structures have different morphologies, discussed more thoroughly in 
the following. It is worth noting that at $T=0.10$ and for large $k_{\mathrm{d}}, e_{\mathrm{m}}$ assumes slightly smaller values (and $b_{\mathrm{m}}$ assumes slightly bigger values) with respect to the rigid case for large $k_{\mathrm{d}}$. In contrast, at the highest temperature considered, $e_{\mathrm{m}}$ can decrease (and $b_{\mathrm{m}}$ can increase) by up to a factor of ten when the angular and radial flexibilities are large. Thus, even in the stiff limit at low temperatures, the percolating structures formed by SFPCs are more inter-connected than their rigid counterpart; this fact is due to the release of the single bond per patch constraint in the present model.

The cluster size distribution of the patches, i.e., the number of patch clusters with size $s$, are reported in Fig. 5 for selected systems at the three temperatures investigated; data are only shown along the main diagonal in the plane of the flexibility parameters. These data provide additional information on how the patch flexibility affects the connectivity of the system; they also shed some light on the extremely large values for $e_{\mathrm{m}}$ and $b_{\mathrm{m}}$ observed in some of the most flexible cases. In the rigid reference system, clusters of patches can be composed of only two patches; however, as soon as the single bonding constraint is released, the size of the clusters can grow, since patches can rearrange around their core, paying an increasingly small energetic penalty on decreasing the flexibility parameters.

At $T=0.10$, a peak in the cluster size distribution of the patches is observed at $s=2$ for $\left(k_{\mathrm{d}}, k_{\theta}\right)=(1000,1000)$ and $(100,100)$; the rest of the patches are mainly in the monomeric state, while a small percentage of them are in clusters of size three. The presence of clusters bigger than size two explains why, even in the stiff limit, $b_{\mathrm{m}}$ is bigger in SFPCs than in the rigid reference system where the constraint of the single bond per patch is implemented. On increasing the patch
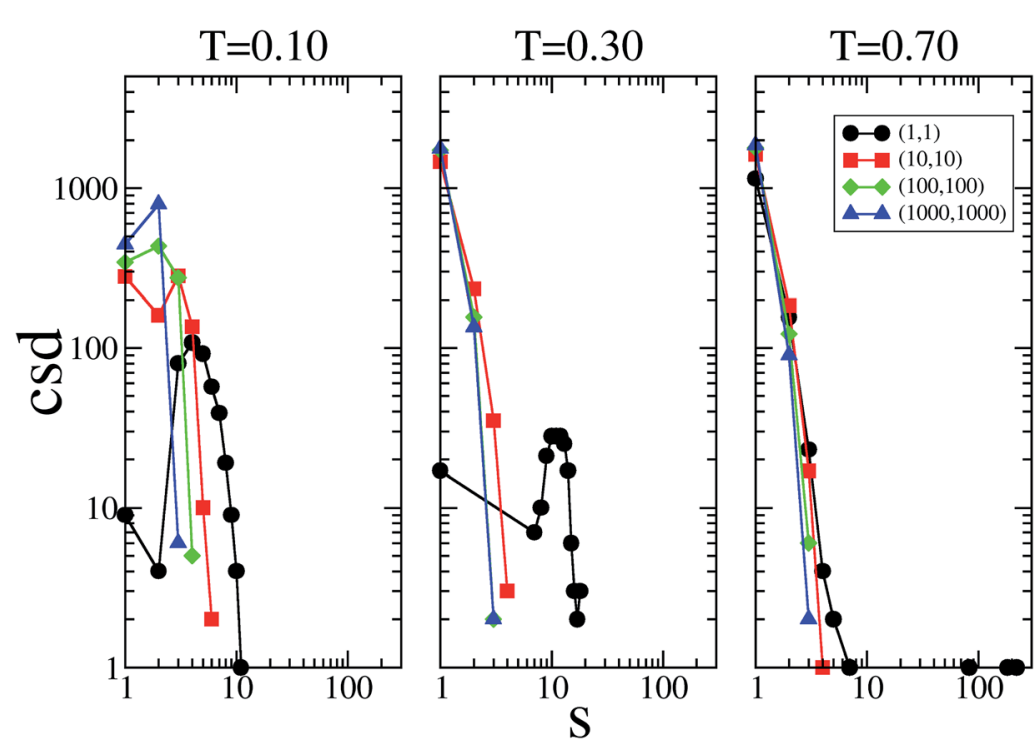

Fig. 5 Cluster size distribution (csd) of the patches, i.e., the number of patch clusters with size $s$, as a function of the cluster size. For the sake of simplicity, data for only four sets of flexibility parameters $k_{\mathrm{d}}$ and $k_{\theta}$ (with $k_{\mathrm{d}}=k_{\theta}$; as labeled) are reported for the three investigated temperature values, namely $T=0.10,0.30$ and 0.70 (from left to right). 
flexibility (i.e., when decreasing $k_{\mathrm{d}}$ and $k_{\theta}$ ), the $s$ corresponding to the peak increases monotonically: to be more specific, when $\left(k_{\mathrm{d}}, k_{\theta}\right)=(10,10), s=3$, while for $\left(k_{\mathrm{d}}, k_{\theta}\right)=(1,1), s=4$. At $T=0.30$ the cluster size distribution peaks at $s=1$ for all systems in the fluid phase; in contrast it has a peak at $s \approx 12$ in the percolating gel network which emerges when $\left(k_{\mathrm{d}}, k_{\theta}\right)=(1,1)$. At $T=0.70$, the cluster size distribution in the fluid systems does not change significantly, while the cluster size of the gel system changes distinctively: while most of the patches are in the monomeric state, big clusters of more than 200 bonds can be found. In the final part of this section, where we select typical configurations of SFPC systems, we further analyze how strongly the morphology of the system is affected by the clustering of the patches.

As already discussed above, the bonding volume of a patch is increased on softening the patch constraints. This increase has an impact not only on the interparticle properties but possibly also on the intra-particle properties. Indeed, the morphology of the percolating network can be affected by the formation of bonds
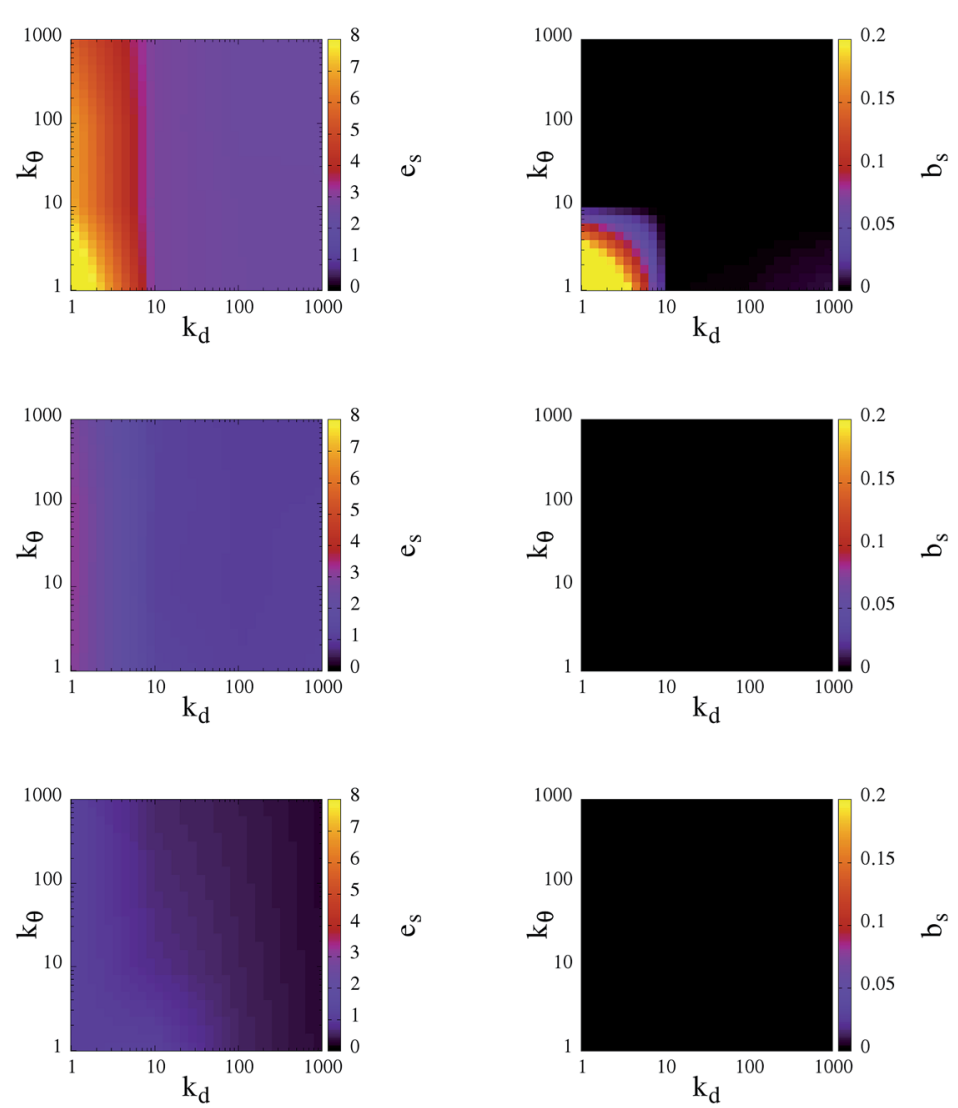

Fig. 6 Left column: intra-particle (self) energy per particle, $e_{s}$; right column: number of intra-particle (self) bonds per particle, $b_{\mathrm{s}}$. Data are reported as contour plots in the $k_{\mathrm{d}} v s . k_{\theta}$ plane for three temperatures: $T=0.70,0.30$ and 0.10 (from top to bottom). Color scales are given at the right-hand sides of the panels. Data, represented by colors, are interpolate between the values on the grid specified by $k_{\mathrm{d}}, k_{\theta}=1,10,100$ and 1000 in their respective reduced units. 
between patches on the same unit. Fig. 6 reports in a contour plot the average intra-particle (or self) energy, $e_{\mathrm{s}}$, and the average number of intra-particle (or self) bonds, $b_{\mathrm{s}}$, at the chosen three temperatures and for all selected systems. Again,
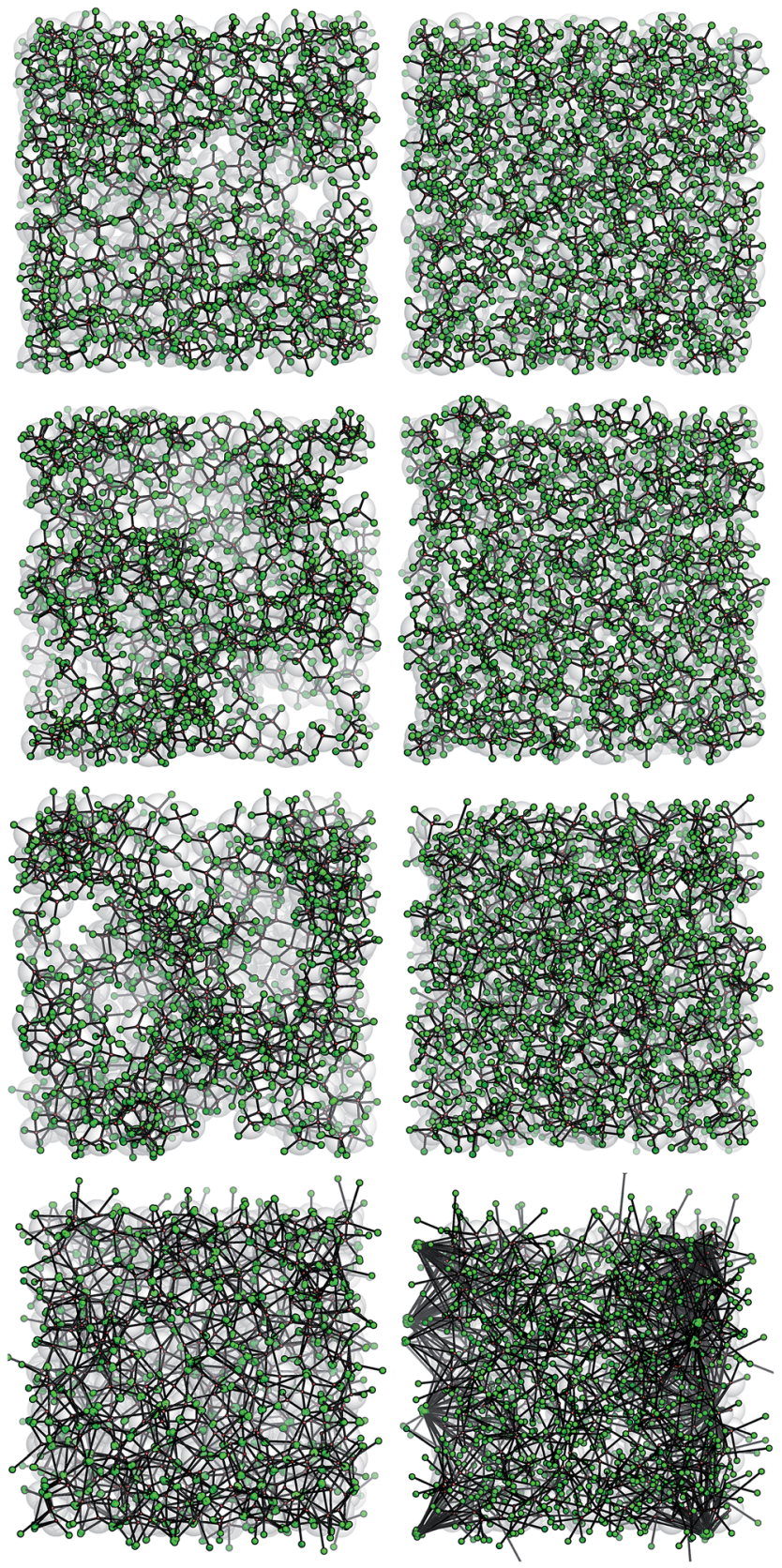

Fig. 7 Snapshots of different systems at two different temperatures: the panels in the left column correspond to $T=0.10$, while the panels in the right column correspond to $T=$ 0.70 . From top to bottom the flexibility parameters change as follows: $\left(k_{d}, k_{\theta}\right)=(1000$, $1000),(100,100),(10,10)$ and $(1,1)$. 
both quantities have a stronger dependence on $k_{\mathrm{d}}$ rather than on $k_{\theta}$ : at a given temperature, $e_{\mathrm{s}}$ and $b_{\mathrm{s}}$ are completely determined by the radial flexibility (quantified by $k_{\mathrm{d}}$ ), which governs whether the system is in the fluid or gel state. In fluid systems no internal bonds are formed and the energy price of the continuously rearranging patches is very close to zero. In contrast, in percolating gels a small percentage of self bonds can occur, according to the angular flexibility of the patches: when $k_{\theta}$ is large, particles do not form internal bonds and hence on an inter-particle level the particles assemble as four coordinated patchy particles; when $k_{\theta}<10$ a small percentage of internal bonds are formed only at the highest temperature analyzed. The energy cost associated with the movements of the patches increases with increasing $T$ and attains a maximum at $T=0.70$ when both $k_{\mathrm{d}}$ and $k_{\theta}$ assume values that are less than 10 . This fact is due to the formation of both inter- and intra-particle bonds: as mentioned before, as a consequence of a deformation of the patch unit, which increases $e_{\mathrm{s}}$, the patches prefer to assemble into big clusters to maximize the number of bonds, thus lowering their $e_{\mathrm{m}}$.

We conclude this section by showing a selection of simulation snapshots of our system in Fig. 7. We report system configurations with $k_{\mathrm{d}}=k_{\theta}$ at $T=0.10$ and 0.70 . As discussed above, at $T=0.10$ all systems form a percolating gel structure, while among the systems at $T=0.70$ only the one with $\left(k_{\mathrm{d}}, k_{\theta}\right)=(1,1)$ is percolating. The fluid systems are in a homogeneous phase characterized by a spatially random distribution of the entities - either patches or cores - in the simulation box; a visual inspection of the panels provides evidence that the respective configurations bear strong similarities with each other (as suggested by the previous quantitative analysis on the single particle structural features). In contrast, the gel structures have different morphologies, as anticipated before. At $T=0.10$ the network in the very stiff limit, i.e., when $k_{\mathrm{d}}, k_{\theta}=(1000,1000)$, is quite homogeneous, resembling the configurations found for the rigid reference case. On increasing the patch flexibility, voids emerge in the gel structure that are related to the formation of clusters with more than two patches. Interestingly, on further increasing the patch flexibility, the percolating network again becomes homogeneous: in striking contrast to the stiff case, the homogeneous gel at $\left(k_{\mathrm{d}}, k_{\theta}\right)$ $=(1,1)$ is characterized by a deeply inter-connected structure. On increasing the temperature, extremely big clusters form in the system. It is worth noting that at $T=0.70$ patches are either unbonded or belong to big clusters; in contrast, at $T=$ 0.30 most of the patches are bonded and belong to relatively smaller clusters.

\section{Conclusions}

Motivated by recent investigations on the self-assembly scenarios of telechelic star polymers (TSPs), ${ }^{21,22}$ we have introduced a simple, soft and flexible patchy colloid (SFPC) model that captures the main features of TSPs in the regime where they behave as complex patchy particles. Introducing a soft core linked to four mobile patches as the building unit of our model, we parameterize the effective interactions between the different entities (either patches or cores) via simple potentials, that are able to capture on a qualitative level the main features of the underlying TSP systems. In addition to conventional Lennard-Jones-type interactions between the different entities, we allow patch displacements in both radial and tangential directions. The Lennard-Jones-type interactions are parameterized to closely reproduce, when the patches are fixed in their 
equilibrium positions, a model with immobile patches which is used as a reference. ${ }^{23}$ The radial and tangential constraints acting on patches belonging to the same particle are parameterized via generalized spring-potentials, whose stiffness is quantified by two respective flexibility parameters. In contrast to the original TSP model, we are able to obtain a deeper insight into the self-organization strategies of SFPCs via systematic variation of the two flexibility parameters. While the density was kept fixed to the value at which the reference system can stabilize a diamond cubic lattice, the temperature (or equivalently the energy interaction strength) was varied over a relatively broad range. All investigations are based on extensive Monte Carlo simulations.

Investigating first the single particle features, i.e., the average distance of the patches from their respective core and the average relative angle between two patches on the same particle, provides clear evidence of the strong impact of the radial flexibility on the former property and of the temperature on the latter property. These features lead to a substantial increase of the effective packing fraction of the system and to an enlarged bonding volume. By analyzing the average inter-particle energy and the average number of bonds between patches belonging to different particles, unambiguous fingerprints can be identified whether a system forms a liquid or a gel phase. Among the latter ones, significantly different morphologies can be identified. Additional insight on the impact of patch flexibility on the connectivity is obtained via a detailed analysis of the cluster size distribution of the patches.

Previous progress in synthesis techniques allowed for the precise and reliable fabrication of star-shaped macromolecules with functionalized end groups. ${ }^{24-26}$ Block copolymer stars have been experimentally analyzed by the group of Fetters ${ }^{27,28}$ in the concentrated density regime; such macromolecules were shown to assemble in structures that depend both on the number of arms and on the percentage of attractive monomers per arm. At the time of the experimental study, no explanation was given about how the functionality of the polymers influences the self-assembly scenario, as the ordered phase was not related directly to the number of arms of each polymer. The theoretical study of ref. 21 and 22 provided an explanation to the experimentally observed phenomena by demonstrating that each single star polymer can undergo a two-stage hierarchical self-assembly: first the polymer stars assemble at the local scale into soft and flexible patchy particles and then they assemble at the global scale into a crystalline structure compatible with the patchiness of the self-aggregated patchy units.

Given the experimental results and the interesting theoretical predictions, the present work aimed at analyzing how the introduction of flexibility into a patchy particle model could affect the behavior of functionalized particle systems. We have presented a very versatile model of a soft and flexible patchy model, that allows to analyze the different types of flexibility independently of each other. The self-assembly scenarios of our model are dictated by the competition between the energy penalties associated with the patch fluctuations and the energy gains due to the patch bonding, giving rise to different self-organization scenarios. Our preliminary results indicate that disordered networks with connectivity properties that can be controlled by tuning the flexibility of the patchy units have come within reach. The design of such disordered architectures will be of high relevance for the development of self-repairing and biomimetic systems. 
It should be noted that systems with related features were recently studied either experimentally or via simulations: (i) in systems where DNA strands were grafted to the liquid interface of emulsions, tuning the valence (via the flexibility of the DNA strands) led to different emulsion architectures, ${ }^{29}$ (ii) in systems of single-stranded DNA-coated colloids tuning the valence (via nonspecific repulsions between the particles) led to different aggregation scenarios. ${ }^{30}$

\section{Acknowledgements}

EB, GK and CNL acknowledge support from the Austrian Science Fund (FWF) under Proj. no. V249-N27 (EB's Elise Richter Grant), P23910-N16, and F41 (SFB ViCoM). BC acknowledges support from the ÖAW, through her APART Fellowship 11723. Computer time at the Vienna Scientific Cluster (VSC) is gratefully acknowledged.

\section{References}

1 G. M. Whitesides and M. Boncheva, Science, 2002, 295, 2418.

2 S. C. Glotzer, Science, 2004, 306, 419-420.

3 A. B. Pawar and I. Kretzschmar, Macromol. Rapid Commun., 2010, 31, 150.

4 E. Bianchi, R. Blaak and C. N. Likos, Phys. Chem. Chem. Phys., 2011, 13, 6397.

5 E. Bianchi, G. Kahl and C. N. Likos, Soft Matter, 2011, 7, 8313.

6 C. E. Snyder, M. Ong and D. Velegol, Soft Matter, 2009, 5, 1263.

7 S. Gangwal, A. Pawar, I. Kretzschmar and O. D. Velev, Soft Matter, 2010, 6, 1413.

8 Q. Chen, S. C. Bae and S. Granick, Nature, 2011, 469, 381.

9 B. Ruzicka, E. Zaccarelli, L. Zulian, R. Angelini, M. Sztucki, A. Moussaïd,

T. Narayanan and F. Sciortino, Nat. Mater., 2011, 10, 56.

10 F. Romano and F. Sciortino, Nat. Mater., 2011, 10, 171.

11 E. Bianchi, C. N. Likos and G. Kahl, ACS Nano, 2013, 7, 4657.

12 E. Bianchi, C. N. Likos and G. Kahl, Nano Lett., 2014, 14, 3412.

13 E. Noya, I. Kolovos, G. Doppelbauer, G. Kahl and E. Bianchi, Soft Matter, 2014, 10, 8464 .

14 F. Smallenburg and F. Sciortino, Nat. Phys., 2013, 9, 554.

15 F. Smallenburg, L. Filion and F. Sciortino, Nat. Phys., 2014, 10, 653.

16 L. Rovigatti, F. Bomboi and F. Sciortino, J. Chem. Phys., 2014, 140, 154903.

17 L. Rovigatti, F. Smallenburg, F. Romano and F. Sciortino, ACS Nano, 2014, 8, 3567.

18 F. Lo Verso, C. N. Likos, C. Mayer and H. Löwen, Phys. Rev. Lett., 2006, 96, 187802.

19 F. Lo Verso, C. N. Likos and H. Löwen, J. Phys. Chem. C, 2007, 111, 15803.

20 F. Lo Verso, A. Z. Panagiotopoulos and C. N. Likos, Phys. Rev. E: Stat., Nonlinear, Soft Matter Phys., 2009, 79, 010401(R).

21 B. Capone, I. Coluzza, F. Lo Verso, C. N. Likos and R. Blaak, Phys. Rev. Lett., 2012, 109, 238301.

22 B. Capone, I. Coluzza, R. Blaak, F. Lo Verso and C. N. Likos, New J. Phys., 2013, 15, 095002.

23 E. Noya, C. Vega, J. P. K. Doye and A. A. Louis, J. Chem. Phys., 2010, 132, 234511.

24 D. Vlassopoulos, T. Pakula, G. Fytas, M. Pitsikalis and N. Hadjichristidis, J. Chem. Phys., 1999, 111, 1760. 
25 M. Pitsikalis, E. Siakali-Kioulafa and N. Hadjichristidis, Macromolecules, 2000, 33, 5460.

26 S. Strandman and H. Tenhu, Polymer, 2007, 48, 7008.

27 D. B. Alward, D. J. Kinning, E. L. Thomas and L. J. Fetters, Macromolecules, 1986, 19, 215.

28 E. L. Thomas, D. B. Alward, D. J. Kinning, D. C. Martin, D. L. Handlin Jr and L. J. Fetters, Macromolecules, 1986, 19, 2197.

29 L. Feng, L.-L. Pontani, R. Dreyfus, P. Chaikin and J. Brujic, Soft Matter, 2013, 9, 9816.

30 S. Angioletti-Uberti, P. Varilly, B. M. Mognetti and D. Frenkel, Phys. Rev. Lett., 2014, 113, 128303. 